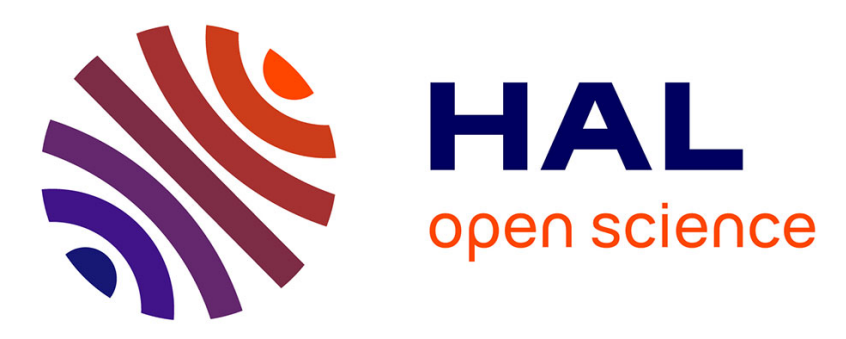

\title{
Nonlinear Wave Phenomena in an Electron-Beam Plasma
}

\author{
K. Yamagiwa, T. Itoh, T. Nakayama
}

\section{To cite this version:}

K. Yamagiwa, T. Itoh, T. Nakayama. Nonlinear Wave Phenomena in an Electron-Beam Plasma. Journal de Physique IV Proceedings, 1997, 07 (C4), pp.C4-413-C4-419. 10.1051/jp4:1997433 . jpa00255589

\section{HAL Id: jpa-00255589 https://hal.science/jpa-00255589}

Submitted on 1 Jan 1997

HAL is a multi-disciplinary open access archive for the deposit and dissemination of scientific research documents, whether they are published or not. The documents may come from teaching and research institutions in France or abroad, or from public or private research centers.
L'archive ouverte pluridisciplinaire HAL, est destinée au dépôt et à la diffusion de documents scientifiques de niveau recherche, publiés ou non, émanant des établissements d'enseignement et de recherche français ou étrangers, des laboratoires publics ou privés. 


\title{
Nonlinear Wave Phenomena in an Electron-Beam Plasma
}

\author{
K. Yamagiwa, T. Itoh and T. Nakayama \\ Department of Physics, Faculty of Science, Shizuoka University, Shizuoka 422, Japan
}

\begin{abstract}
An experiment has been carried out to investigate one-dimensional nonlinear evolution and formation of the localized structure of unstable waves in an electron-beam plasma. The plasma system is linearly unstable against electrostatic perturbations of frequencies lower than the critical frequency $\omega_{\circ},\left(\fallingdotseq \omega_{\mathrm{p} e}\right.$, plasma frequency), but is stabilized in its nonlinear stage by generating wave packets irregularly. The amplitude of each wave packet never grows infinitely. The average time width of the packets is inversely proportional to the linear growth rate $\gamma$, where $\gamma \propto\left(n_{\mathrm{b}} / n_{0}\right)^{1 / 3} \omega_{\mathrm{p}}$ ( $n_{\mathrm{b}}$ : beam density, $n_{0}$ : plasma density ). The system is also unstable for an external rf-burst with a narrow time-width, and then finally becomes stable by the emission of a series of burst waves. The number of emitted bursts increases with the beam density.
\end{abstract}

\section{INTRODUCTION}

Since the pioneering work on nonlinear phenomena of Langmuir waves was done by Zakharov [1], considerable progress has been made in this area [2]. There have also been growing interests [3-8] on nonlinear wave phenomena of unstable modes. Wong and Cheung [3] experimentally investigated nonlinear evolution and collapse of the electron-beam-instability. They explained their observed results with the theory of three dimensional Langmuir collapse in which the beam effect is not included.

The dispersion properties of unstable modes [4-9], however, are quite different from stable ones. Intrator et al. [4] observed unstable electron-beam wave packets. They tentatively explained their experimental results by a nonlinear geometrical optics theory, not by Zakharov Langmuir-wave collapse. N. Yajima and Tanaka [5] predicted the existence of soliton modes of unstable electron-beam waves which are described by a nonlinear Schrödinger equation with the beam correction. T. Yajima and Wadati [6] also theoretically showed that solitons arise as a result of the competition between instability and nonlinearity in unstable systems. We [7] have studied nonlinear evolution of one-dimensional perturbations in a weak electron-beam plasma $\left(n_{\mathrm{b}} / n_{0}<0.1 \%\right)$. Then we found that the initial growth of unstable modes and the formation of soliton-like excitation are characterized by the spatial linear growth rate $k_{i}$. In the case of a medium electron-beam $\left(0.5 \%>n_{\mathrm{b}} / n_{0} \geqq 0.1 \%\right)[8]$, beam modes were initially unstable against frequencies lower than $\omega_{c}$, and finally they were stabilized by generating nonlinear wave packets. The characteristic time for formation of the structure was much shorter than the ion response time $\left(\fallingdotseq 1 / \omega_{p \mathrm{i}}\right.$ : inverse of ion plasma frequency $)$.

In this paper we will present our recent observations on nonlinear wave phenomena in an electron-beam plasma and discuss them with theories on the soliton modes in unstable systems.

\section{PLASMA DEVICE AND EXPERIMENTAL SETUP}

A target plasma is produced by a dc discharge in a so called multi-dipole plasma device shown in Fig. 1(a). An additional magnetic field $(=90 \mathrm{G})$ is externally applied parallel to the axis of the plasma chamber to observe the one-dimensional behavior of the beam modes. The working pressure of $\mathrm{Ar}$ gas for the discharge is adjusted to $1 \times 10^{-5}$ Torr to reduce collisional loss of beam electrons with neutral particles. We inject a pulse electron beam with duration of $7 \times 10^{-6}$ s into a target plasma along the external magnetic field. Figure $1(\mathrm{~b})$ shows the experimental setup. The beam current $I_{\mathrm{b}}$ passing through the plasma is measured by using a collector located at the opposite end from the beam gun. 
(a)

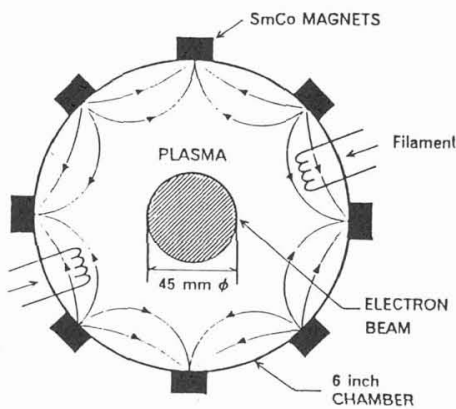

(b)

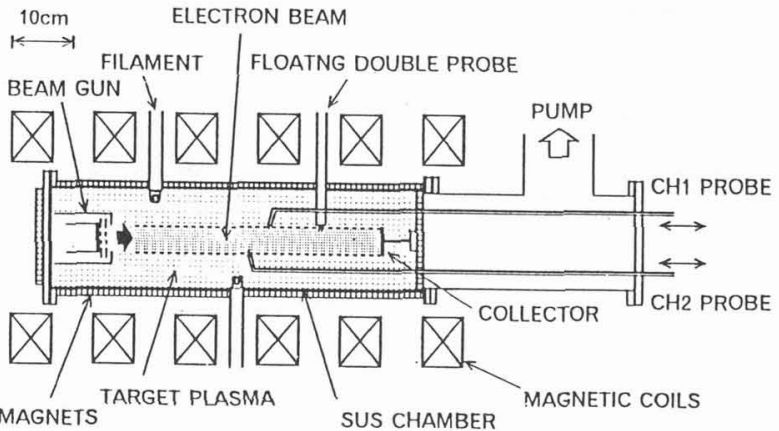

Figure 1: (a) Electron-beam plasma device. (b) Experimental setup.

Density fluctuations $\tilde{n}(t)$ are picked up with two small plane probes (Mo disks of $3 \mathrm{~mm}$ in diameter) located at different positions, $z_{1}$ and $z_{2}$, measured from the beam gun and then two sets of realtime data are captured by a fast digitizing oscilloscope (HP54510A, 1GSa/s, 2ch, 8kW/ch). The maximum amplitude of the density fluctuations normalized by the equilibrium plasma density, $\tilde{n}(t) / n_{0}$, seems to be over ten percent in the present experiment. However we find it difficult to determine the exact value of it because of the high frequency $\left(\omega \doteqdot \omega_{p}\right.$.). So that we will show the data of $\tilde{n}(t)$ in relative units. Typical experimental parameters are summarized in Table 1.

Table 1. Experimental parameters

\begin{tabular}{lll}
\hline \hline Working pressure (Ar) & $p$ & $=1 \times 10^{-5} \mathrm{Torr}$ \\
Plasma density & $n_{0}$ & $=3 \times 10^{14} \mathrm{~m}^{-3}$ \\
Electron ternperature & $T_{t}$ & $=3 \sim 4 \mathrm{eV}$ \\
Plasma frequency & $\omega_{0_{0}} / 2 \pi$ & $=150 \sim 160 \mathrm{MHz}$ \\
Debye length & $\lambda_{0}$ & $=0.9 \mathrm{~mm}$ \\
Bean density & $n_{b} / n_{0}$ & $=0.02 \sim 0.5 \%$ \\
Beam velocity & $v_{b} / v_{t}$ & $=4 \sim 6($ thermal velocity of electrons) \\
Unstable wavenumber & $k \lambda_{0}$ & $=0.2$ (typical value) \\
Beam diameter & $2 n_{\mathrm{o}}$ & $=45 \mathrm{~mm}(\mathrm{kr}=7)$ \\
Beam duration & $t$ & $=7 \times 10^{-6} \mathrm{~s}\left(\omega_{\mathrm{Di}} t=20\right)$ \\
System length for beam path & $t$ & $=50 \mathrm{~cm}$ \\
\hline
\end{tabular}

\section{EXPERIMENTAL RESULTS}

\subsection{Fundamental properties of unstable beam modes}

When an electron beam is injected into the target plasma, unstable noise patterns are initially excited along the beam path and the rms-density fluctuation $\left\langle\tilde{n}(t) / n_{0}\right\rangle_{\mathrm{rms}}$ grows to several percent in the

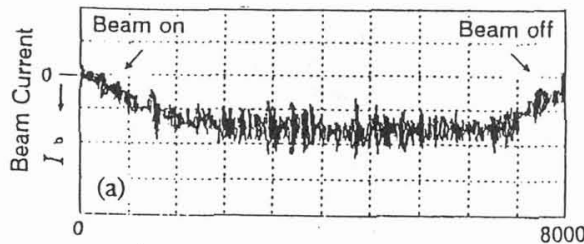

$n_{\mathrm{b}} / n_{0}=0.15 \%$ and $\mathrm{vo}_{\mathrm{o}} / \mathrm{T}=5$
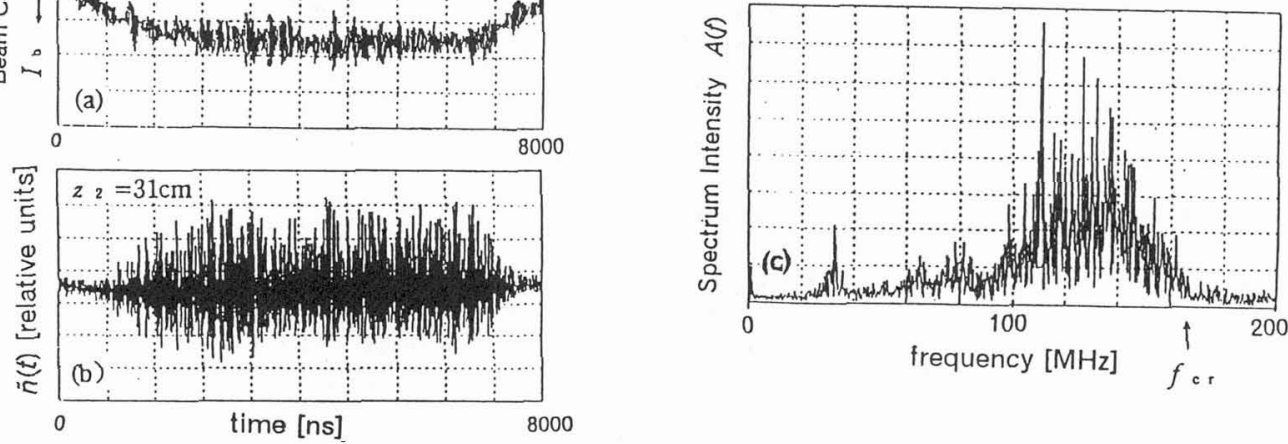

Figure 2: (a) Beain current $I_{\circ}(t)$, (b) density fluctuations $\tilde{n}(t)$ and (c) Fourier spectnum $A(t)$ of $\tilde{n}(t)$. 
downstream region. Finally the system becomes stable in its nonlinear stage by generating nonlinear wave packets. Typical data of the beam current $I \mathrm{~b}(t)$, density fluctuations $\tilde{n}(t)$ and its Fourier spectrum $A(f)$ are shown in Figs. 2(a), (b) and (c), where beam density $n_{0} / n_{0}=0.15 \%$, beam velocity $v_{0} / v_{\mathrm{T}}=5$ and the probe position $z_{2}=3.1 \mathrm{~cm}$. The critical frequency $f_{\mathrm{c}}$, indicated by an arrow in Fig. 2(c) is nearly equal to the plasma frequency $f_{\mathrm{pc}}(\fallingdotseq 160 \mathrm{MHz})$. The plasma system should be unstable in a frequency range lower than the critical frequency $f_{\mathrm{er}}$.

In order to observe evolution of unstable beam waves we repetitively inject pulse electron beams and simultaneously apply small rf-signals as initial perturbations to the control grid of the beam gun. The spatial development of unstable waves is measured by an interferometry method, with a boxcar integrator as a low pass filter. In the initial stage of propagation, excited beam waves are linearly unstable, that is, their amplitudes exponentially increase along the beam path, but saturate in the downstream region. Finally a symmetric envelope $\left(\propto k_{i} \operatorname{sech}\left[k_{i}\left(z-z_{0}\right)\right]\right)$ is produced [7]. The sets of dispersion data extracted from the linear phase are plotted with closed circles in Fig. 3.

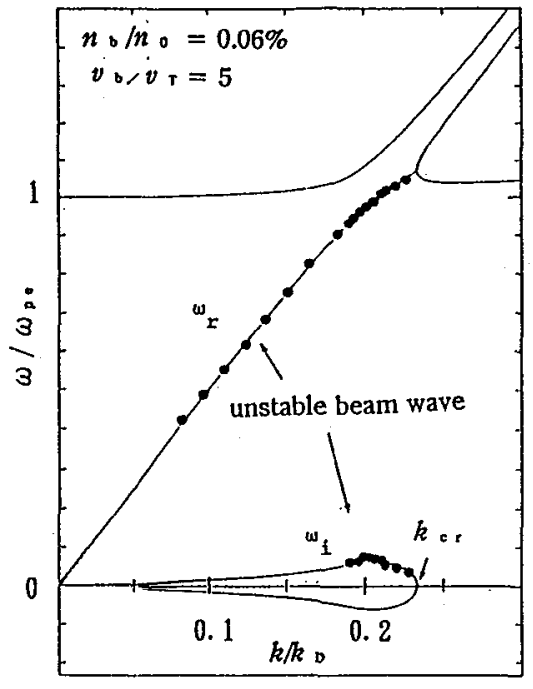

Figure 3: Dispersion relation. Closed circles: Experimental data in the linear phase. Solid curves: Calculated from eq.(1).

The temporal growth rate $\omega_{i}$ is given by the product $\left|k_{i} v_{s}\right|$, where $k_{i}$ is the spatial growth rate in the linear stage and $v_{\mathbf{g}}$ is the group velocity. Solid curves indicate the dispersion relation for linear waves in an electron-beam plasma given by [9]

$$
1=-\frac{\omega_{p e}^{2}}{\left(\omega^{2}-k^{2} v_{\mathrm{T}}^{2}\right)}-\frac{\omega_{\mathrm{pb}}^{2}}{\left(\omega-k v_{\mathrm{b}}\right)^{2}}
$$

The equation has a pair of complex conjugate roots, $\omega_{\mathrm{r}} \pm i \omega_{\mathrm{i}}$, for a fixed wave number $k$.less than the critical value $k_{\circ}\left(\fallingdotseq \omega_{\mathrm{p}} / \nu_{\mathrm{b}}\right)$. One of these roots, $\omega_{r}+i \omega_{i}$ corresponds to unstable modes. Experimental sets of data agree very well with these unstable modes. This dispersion relation also well explains the behavior of self-excited beam modes in the linear phase as shown below.

\subsection{Nonlinear evolution and stabilization of beam modes: formation of nonlinear wave packets}

Density fluctuations in Fig. 2(b) appear very spiky when viewed on the oscilloscope. Similar data sets are observed at $z_{1}=28 \mathrm{~cm}$. In Fig. 4 , both data sets of the density fluctuations detected at $28 \mathrm{~cm}$ and $31 \mathrm{~cm}$ are magnified by 16 times in time scale on the horizontal axis. It is found that (1) irregular wave packets with a narrow width shorter than $20 \mathrm{~ns}$ are generated, (2) wave packets at $z_{2}=31 \mathrm{~cm}$ are delayed by $12 \mathrm{~ns}$ compared with ones at $z_{1}=28 \mathrm{~cm}$, that is, they propagate downstream with the group velocity $v_{\mathrm{g}}=2.5 \times 10^{6} \mathrm{~m} / \mathrm{s}$ and (3) the phase velocity $v_{\phi}=3.7 \times 10^{6} \mathrm{~m} / \mathrm{s}$ (calculated from the cross-correlation between both realtime sets of data). The facts of (2) and (3) are consistent with the dispersion relation of unstable modes in the electron-beam plasma shown in Fig. 3. However we find it very difficult to obtain the time-width and amplitude of those wave packets because of their irregularity. 


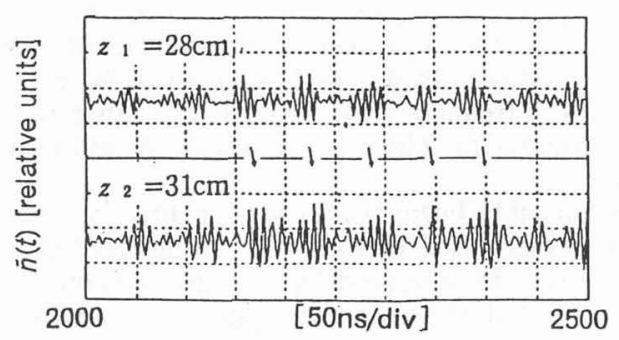

Figure 4: Density fluctuations $\tilde{h}(t)$ at $z_{1}=28 \mathrm{~cm}$ and $z_{2}=31 \mathrm{~cm}$, where $m_{\mathrm{b}} / n_{0}=0.15 \%$ and $v_{\mathrm{b}} / v_{\mathrm{T}}=5: t=2000 \sim 2500 \mathrm{~ns}$

Figures $5(\mathrm{a})$ and $5(\mathrm{~b})$ show realtime data $\tilde{n}(t)$ and self-correlation $c(t)$, respectively. We can estimate the average value of the time-width $\Delta t$ by calculating the correlation time $\Delta \tau_{c}$. In this case, the correlation time $\Delta \tau_{0}=21.5 \mathrm{~ns}$ corresponds to the average time-width $\Delta t$ of the wave packets. With this method we tried to measure the average value of $\partial t$ varying the beam density $n_{0} / n_{0}$.

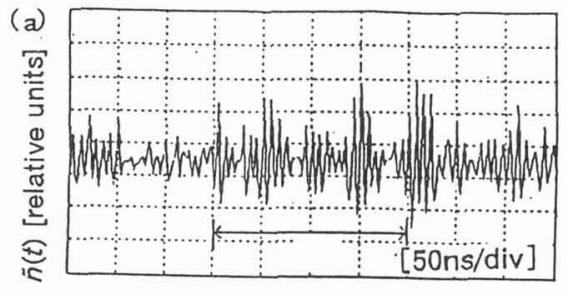

time

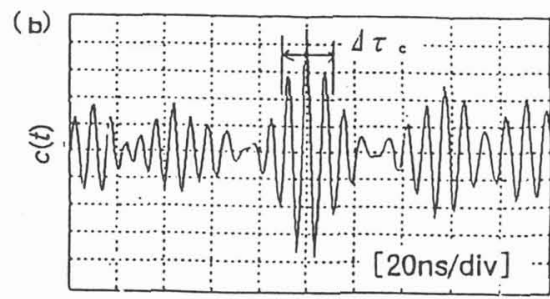

time

Figure 5: (a) Realtime data $\tilde{n}(t)$ and (b) self-correlation $c(t): n_{\mathrm{b}} / n_{0}=0.12 \%, v_{\mathrm{b}} / v_{\mathrm{r}}=5$.

In Fig. 6, experimental values of $\Delta t$ are plotted on a logarithmic graph with closed circles as a function of beam density $n_{\mathrm{b}} / n_{0}(=0.10 \sim 0.45 \%)$. In this graph we also draw the best fit line with a gradient of $-1 / 3$. Thus we find the empirical relation given by

$$
\Delta t \propto\left(n_{\circ} / n_{0}\right)^{-1 / 3}
$$

It is well known [9] that the linear growth rate $\gamma$ of the beam modes is proportional to $\left(\mathrm{n}_{\mathrm{t}} / \mathrm{n}_{0}\right)^{1 / 3}$ and the maximum growth rate $\gamma_{m a x}$ is given by $3^{1 / 2} 2^{-4 / 3}\left(n d n_{0}\right)^{1 / 3} \cdot \omega_{p e}$ for $\omega^{\circ}=\omega_{\text {pe }}$. Observed values $\Delta t(=10$ $\sim 20 \mathrm{~ns})$ are proportional to $\gamma^{-1}$, and their magnitudes are 2 times $\left(\gamma_{\max }\right)^{-1}$. This indicates that the structure of the nonlinear wave packets is determined by the linear growth rate.

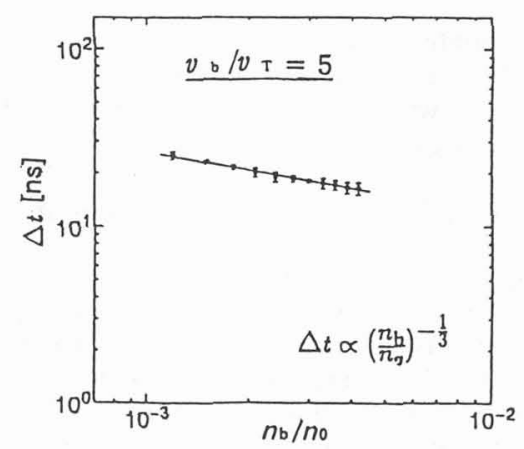

Figure 6: Average time-width $\Delta t$ of wave packets as a function of beam density $n_{\mathrm{b}} / n_{0}$. 


\subsection{Evolution of a single rf-burst and stabilization by the emission of bursts}

We present interesting results of the test wave experiment. When a small rf-burst signal with the time width of $50 \mathrm{~ns}$ as an initial perturbation is applied to the control grid of the beam gun, a single burst wave is excited from the noise level. This burst wave initially grows linearly along the electron stream and the amplitude of the unstable burst gradually saturates in its nonlinear stage. Finally the system becomes stable by the emission of a series of burst waves. In Figs. 7(a), (b) and (c) we show how a single if-burst grows, saturates and generates new burst waves. In the case of a weak-beam injection ( $n_{b} / n_{0}=0.03 \%$ ) a single burst wave only grows from noise level along the beam path. In this case

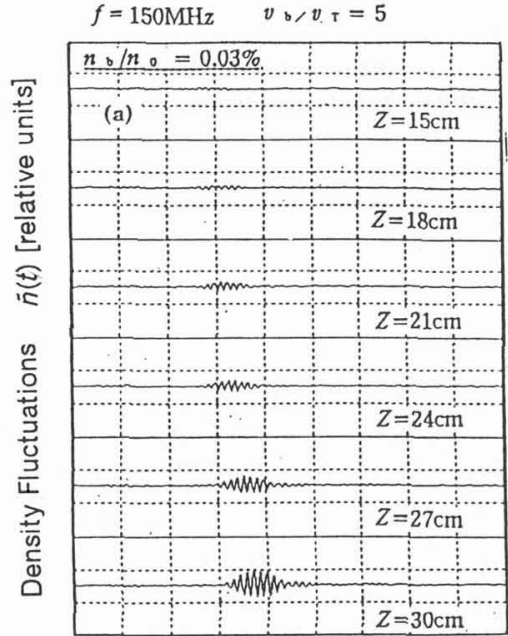

[50ns/div]

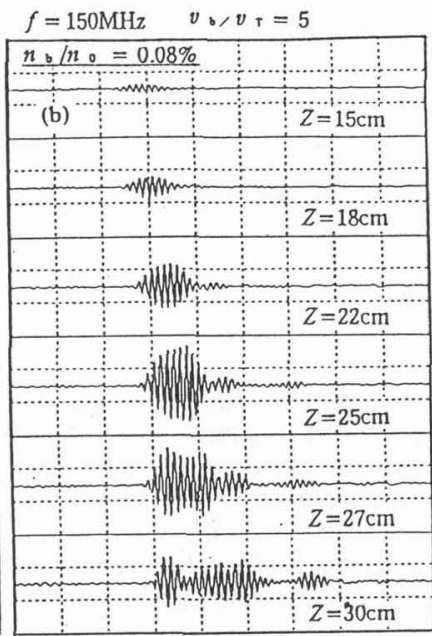

[50ns/div]

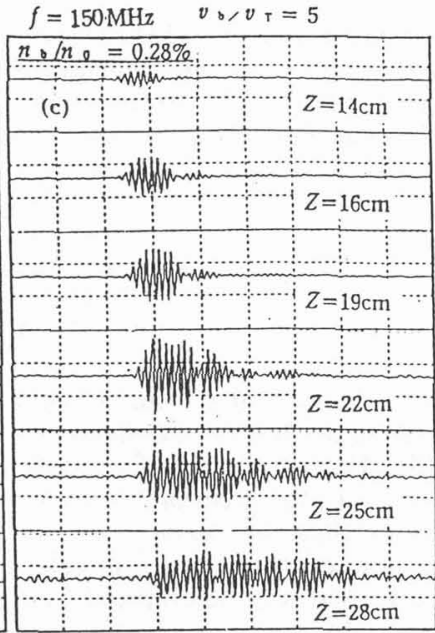

[50ns/div]

Figure 7: Nonlinear evolution of a single burst wave and the emission of a series of burst waves. (a) $n_{\mathrm{b}} / n_{0}=0.03 \%$, (b) $n_{\mathrm{b}} / n_{0}=0.08 \%$, (c) $n_{\mathrm{b}} / n_{0}=0.28 \%$.

nonlinear saturation is very rare. Qn the other hand, in the case of a medium beam injection $\left(n_{\mathrm{b}} / n_{0} \geqq\right.$ $0.05 \%$ ), a single burst wave initially grows linearly along the electron stream and the amplitude of the

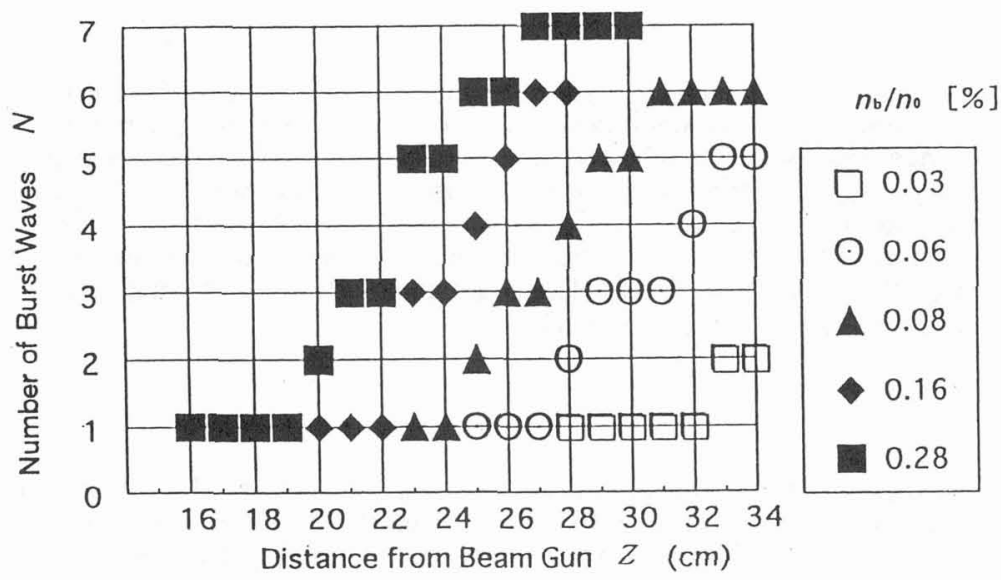

Figure 8: Number of burst waves as a function of the distance from beam gun, for several values of $n_{\mathrm{b}} / n_{0}$. 
unstable burst gradually saturates in its nonlinear stage. Finally the system becomes stable by the emission of a series of burst waves along the beam path as shown in Figs. 7(b) and (c). Ion motion has no effect on the phenomena because the ion response time $\left(1 / \omega_{p i} \stackrel{ }{=} 300 \mathrm{~ns}\right)$ is longer than the observed time scale $(10 \sim 200 \mathrm{~ns})$.

Figure 8 shows the number $N$ of emitted burst waves as a function of probe position $z$, the distance from the beam gun, where the beam density $n_{\mathrm{b}} / n_{0}$ is varied from 0.03 to $0.28 \%$. It is noticeable that the number of emitted bursts increases with the beam density $n_{\mathrm{b}} / n_{0}$ and the electron-beam plasma system becomes stable by emitting a series of burst waves. Therefore it never remains unstable for an infinite period of time.

\section{DISCUSSION}

N. Yajima and M. Tanaka [5] theoretically studied nonlinear evolution of unstable electron beam waves in an electron beam plasma. When the beam velocity $\nu_{\mathrm{b}}$ is sufficiently large compared with the thermal velocity of electrons $v_{\text {r, }}$ the system is governed by a nonlinear Schrödinger equation with an additional term of beam correction ( the right hand side of the first equation ):

$$
\begin{aligned}
& \left(i \partial_{1}-1+\partial_{z}{ }^{2} / 2+|f|^{2}\right) f=-\left[\left(n_{\mathrm{b}} / n_{0}\right) / 2\right] g \\
& \left(\partial_{1}+v_{2}\right)^{2} g=f \\
& ; E=4\left(2 \pi n_{0} T\right)^{1 / 2} f, \quad n_{\mathrm{b}}=2^{3 / 2} \cdot n_{\mathrm{b}} \partial_{2} g, \quad v=v_{\mathrm{b}} / v_{\mathrm{T}}
\end{aligned}
$$

They showed that the system is linearly unstable against electrostatic perturbations of large wavelengths but is stabilized in its nonlinear stage due to the ponderomotive force of high frequency fields. The amplitude of an unstable wave behaves in proportion to $\gamma \operatorname{sech}(\gamma t)$ as a function of time $t$, where $\gamma$ is the temporal linear growth rate at $k \fallingdotseq k_{\text {.r }}$ (critical wave number $\fallingdotseq \omega_{\mathrm{pe}} / \nu_{\mathrm{b}}$ ). They also showed that the plane wave is unstable for long wavelength modulation and is stabilized by the emission of a series of envelope solitons. Their theoretical study predicts that the structure of the soliton modes is determined by the initial growth rate but not by the amplitude of initial perturbations, as in stable systems. T. Yajima and M. Wadati [6] also showed that solitons arise as a result of the competition between instability and nonlinearity.

The present experiment showed that the unstable modes with localized structures are soliton-like excitations. They can exist in an electron-beam plasma, which is a weakly dispersive medium. Their dynamical processes are developed differently from the usual soliton processes in which the number of solitons and their identities are conserved. These soliton-like localized modes are completely different from Zakhalov's Langmuir solitons which arise in a rather strong dispersive medium. The present observations shown in Figs. 4, 6, 7 and 8 are qualitatively in agreement with their theoretical predictions $[5,6]$.

\section{CONCLUSIONS}

The electron-beam plasma is linearly unstable in a frequency range lower than the critical frequency $\left(\doteqdot \omega_{\mathrm{p}}\right)$. The beam plasma system is stabilized in its nonlinear stage by generating nonlinear wave packets, the average space-time width of which is inversely proportional to the linear growth rate $\gamma(\alpha$ $\left.\left(n \mathrm{~d} / n_{0}\right)^{1 / 3}\right)$. The system is also linearly unstable for a single if-burst wave and becomes stable by emission of burst waves along the beam path. The number of emitted burst waves increases with the beam density $n \mathrm{~b} / n_{0}$. The results indicate that the nonlinear structures of the unstable electron-beam waves are determined by the linear growth rate. The characteristic time for formation of the structure is much shorter than the ion response time $\left(\fallingdotseq 1 / \omega_{\mathrm{p}}\right)$.

\section{Acknowledgments}

The authors gratefully acknowledge valuable discussions of Professor M. Tanaka of NIFS, Toki (JAPAN), stimulating discussions of Professor M. Wadati and Dr. T. Yajima of Tokyo University and careful observations of Mr. H. Takeshita (Msc) and Miss K. Hirahama (Msc) for this work. They also thank Mr. Nick Brown of Sussex University for his kind inspection of the English of this paper. The present work was partially supported by a Grand-in-Aid for Scientific Research from the Ministry of Education. 


\section{References}

[1] Zakharov V. E., Sov. Phys. JETP 35 (1972) 908.

[2] Goldman M. V., Rev. Mod. Phys. 56 (1984) 709.

[3] Wong A. Y. and Cheung P. Y., Phys. Rev. Lett. 52 (1984) 1222.

Cheung P. Y. and Wong A. Y., Phys. Fluids 28 (1985) 1538.

[4] Intrator T., Chan C., Hershkowitz N. and Diebold D., Phys. Rev. Lett. 53 (1984) 1233.

[5] Yajima N. and Tanaka M., Prog. Theor. Phys. Suppl. No.94 (1988) 138.

[6] Yajima T. and Wadati M., J. Phys. Soc. Jpn. 59 (1990) 41.

Yajima T. and Wadati M., J. Phys. Soc. Jpn. 59 (1990) 3237.

[7] Yamagiwa K., Mieno T. and Tanaka M., Proc. 1992-ICPP (Innsbruck, 1992) Part III 1859.

[8] Yamagiwa K., Tanaka M., Itoh T. and Nakayama T., Proc. 1996-ICPP (Nagoya, 1996) Vol. 1774. Yamagiwa K., Itoh T. and Nakayama T., Proc. PFNL-96(Sendai,1996), to be published in "Double Layers-Potential Formation and Related Nonlinear Phenomena in Plasmas" (World Scientific Publishing Co., Singapore) p.407.

[9] Briggs R. J., "Electron-Stream Interaction with Plasmas" in Research Monograph No. 29 (M.I.T. Press, Cambridge, 1964) and "Two-Stream Instabilities" in Advance in Plasma Physics, Edited by A. Simon and W. B. Thompson (Interscience Publisher, New York, 1971) Vol. 4, p.43. 